\title{
Unusual Properties of Enhancement Factor in Open Wave Chaotic System with Time-Reversal-Invariance Violation
}

\author{
M. Biatous ${ }^{a}$, B. Dietz ${ }^{b}$ And L. SiRKo ${ }^{a, *}$ \\ ${ }^{a}$ Institute of Physics, Polish Academy of Sciences, \\ Aleja Lotnikow 32/46, PL-02668 Warsaw, Poland \\ ${ }^{b}$ Lanzhou Center for Theoretical Physics \\ and the Gansu Provincial Key Laboratory of Theoretical Physics, \\ Lanzhou University, Lanzhou, Gansu 730000, China \\ Doi: $10.12693 /$ APhysPolA.139.462 \\ *e-mail: sirko@ifpan.edu.pl
}

\begin{abstract}
We discuss experimental investigations showing that above a certain size of time-reversal $(\mathcal{T})$ invariance violation the increase of the openness of a wave chaotic system can lead to an increase of the elastic enhancement factor, that is, an enhancement of scattering in backward direction. In the experiment, a quantum billiard with partially violated time-reversal invariance, characterized by the $\mathcal{T}$-invariance violation parameter $\xi$, which varies between zero for preserved and one for completely violated $\mathcal{T}$-invariance, is simulated with a flat quarter-bow-tie microwave cavity which contains two cylindrical ferrites that are magnetized by an external magnetic field. The elastic enhancement factor $F_{M}(\eta, \gamma, \xi)$ is investigated as a function of internal absorption and openness. Particularly, we focus on the frequency range of strongest $\mathcal{T}$-invariance violation where the increase of the number of open channels causes a boost of the elastic enhancement factor $F_{M}(\eta, \gamma, \xi)$, instead of the expected lowering. Our study extends our recently published investigation [Phys. Rev. E 102, 042206 (2020)] towards a higher number of open channels and therefore larger openness of the system.
\end{abstract}

topics: wave chaos, microwave billiards, time-reversal invariance violation, quantum chaotic scattering

\section{Introduction}

According to the Bohigas-Giannoni-Schmit conjecture [1], the features of quantum systems with a chaotic classical dynamics are reflected in the fluctuation properties in their eigenvalue spectra and coincide with those of random Hermitian matrices with Gaussian distributed entries of zero mean belonging to one of the three fundamental symmetry classes, namely the orthogonal, unitary, or symplectic one characterized by the parameter $\beta=1,2,4$, respectively [2]. This conjecture was verified by numerous experimental and numerical studies. Typical physical systems with preserved time-reversal $(\mathcal{T})$ invariance and a fully chaotic classical dynamics exhibit the statistics of real-symmetric random matrices from the Gaussian orthogonal ensemble (GOE). Some examples of such systems are quantum wells [3], molecular spectra [4], microwave flat billiards [5] and microwave networks [6, 7] which are most suitable for the experimental verification of random matrix theory (RMT) predictions. The spectral properties of random matrices from the Gaussian unitary ensemble (GUE) are observed in systems with violated $\mathcal{T}$-invariance, for example in atoms in a constant external field [8], graphene quantum dots [9], the Rydberg excitons [10], nuclear reactions $[11,12]$, microwave billiards $[13,14]$ and microwave networks $[15,16]$. Random matrix theory was also successfully applied to systems with partially broken time reversal symmetry [17, 18]. These studies have become an inspiration for the experimental analysis of physical systems that exhibit the transition from GOE and GUE behaviour [19, 20]. The transition from Poissonian level statistics exhibited by classically integrable systems to GOE or GUE statistics leading to level correlations was also studied [21-25].

The specific features of the complicated scattering systems belonging to different symmetry classes can be characterized quantitatively by the elastic enhancement factor $F_{M}^{(\beta)}(\eta, \gamma)$, which is expressed as the ratio of the elastic and inelastic scattering $S$-matrix autocorrelation coefficients [26, 27]. It was introduced by Moldauer [28] and investigated in nuclear physics [29-32]. Its universality was thoroughly studied theoretically [30, 33] and experimentally in various systems, including microwave cavities [5, 19, 27, 34-46] and microwave networks simulating chaotic quantum graphs with preserved and violated time reversal symmetry $[6,16,47-52]$. The above simulators are complemented by experiments 
with the Rydberg atoms strongly driven by microwave fields [53-65]. The enhancement factor depends on the openness $\eta=M T$ of a chaotic system $[31,32]$, where $M$ is the number of identical scattering channels $i$. In turn, $T=\frac{1}{M} \sum_{i=1}^{M} T_{i}$ is the average of the transmission coefficients for $T_{i}=1-\left|\left\langle S_{i i}\right\rangle\right|^{2}$ with $\left\langle S_{i i}\right\rangle$ denoting the average elastic $S$-matrix [31, 32]. The transmission coefficients provide a measure for the size of the coupling of the scattering channels to the interior states. The enhancement factor $F_{M}^{(\beta)}(\eta, \gamma)$ also depends on the size of the internal absorption $\gamma=2 \pi \Gamma / \Delta$. Here, $\Delta$ denotes the mean resonance spacing and $\Gamma$ is the average resonance width, which in the experiments with a microwave cavity consists of the internal absorption width $\Gamma_{\text {cav }}$ and the width $\Gamma_{\text {esc }}$ due to the impact of additional open channels which describe the coupling between the scattering channels $i$, that is, the antennas and the interior region.

In this paper, we extend our experimental study [20] towards larger openness $\eta=M T$, with $M=12$, thereby confirming that the increase of the openness of a microwave cavity with partially violated $\mathcal{T}$-invariance can lead to an increase of the enhancement factor.

\section{Experimental setup}

In the experiment, a fully chaotic cavity (see Fig. 1) with the shape of a quarter-bowtie billiard was used. The area and the perimeter of the cavity are $A=1828.5 \pm 5.0 \mathrm{~cm}^{2}$ and $L=202.3 \pm 2.0 \mathrm{~cm}$. The cavity is made of two plates of polished aluminum type EN 5754 which are squeezed tightly together with 127 screws at a $2 \mathrm{~cm}$ distance. The basin of the cavity was milled out of the bottom plate. The height of the cavity equals $h=1.2 \mathrm{~cm}$ corresponding to a cut-off frequency of $\nu_{\max }=c /(2 d) \simeq 12.49 \mathrm{GHz}$, where $c$ is the speed of light in vacuum. We considered microwave frequencies below $\nu_{\max }$ so that only transverse magnetic modes were excited inside the cavity. Then, the Helmholtz equation describing the microwave cavity and the Schrödinger equation for the corresponding quantum billiard are mathematically equivalent. The inner surface of the cavity is covered with a $20 \mu \mathrm{m}$ layer of silver to reduce the internal absorption. The top lid of the cavity contains 12 randomly distributed identical antenna holes marked from 1 to 12 in Fig. 1a. The two antennas 1 and 2 with lengths of $5.8 \mathrm{~mm}$ and pin diameter of $0.9 \mathrm{~mm}$ play the role of the scattering channels coupled to an Agilent E8364B vector network analyzer (VNA). More open channels $3 \leq M \leq 12$ were realized by attaching to the cavity $M-2$ additional antennas of the same size but shunted with $50 \Omega$ loads. Thereby, the openness of the cavity was gradually increased.

All measurements were done in the frequency range $\nu=6-12 \mathrm{GHz}$, where the internal absorption takes values between $6<\gamma<20$. In this frequency
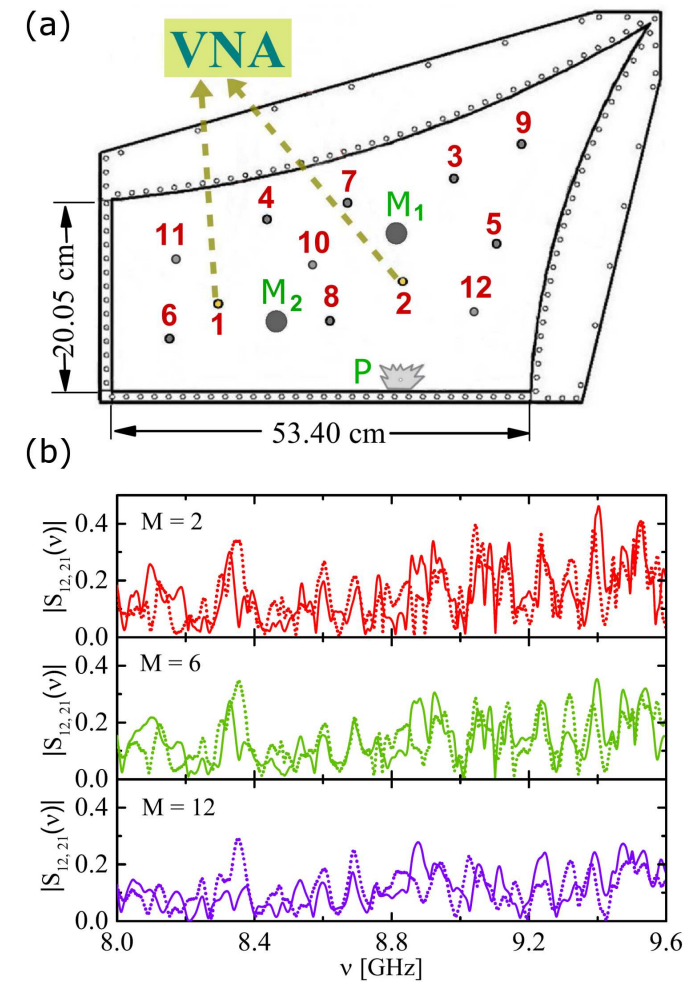

Fig. 1. (a) The experimental setup. The vector network analyzer Agilent E8364B was connected to the microwave antennas No. 1 and 2 with flexible microwave cables. The top lid of the microwave billiard contains 12 randomly distributed holes. Antennas of the same size were protruded through these holes. Two of them were used as measuring antennas while the others were shunted with $50 \Omega$ loads and represent additional open channels. The $\mathcal{T}$-invariance violation was induced by two pieces of ferrite inserted into the cavity and magnetized by external magnets $\mathrm{M}_{1}$ and $\mathrm{M}_{2}$ placed above and below the cavity. A metallic perturber $P$ was moved inside the cavity alongside its walls in order to create different realizations of the billiard. In part (b), we show examples for $\left|S_{12}(\nu)\right|$ (full line) and $\left|S_{21}(\nu)\right|$ (dotted line) measured in the frequency range $\nu=8.0-9.7 \mathrm{GHz}$ for $M=2, M=6$, and $M=12$ open channels, respectively.

range, the wavelength is small as compared to the cavity dimension, implying that it is within the semiclassical regime. A metallic perturber with perimeter $L \simeq 26 \mathrm{~cm}$ and area $A \simeq 9 \mathrm{~cm}^{2}$ was placed inside the cavity and moved along the vertical walls. This enabled to create 100 realizations of a wave-chaotic cavity. In order to measure the two-port scattering matrix

$$
\hat{S}(\nu)=\left(\begin{array}{ll}
S_{11}(\nu) & S_{12}(\nu) \\
S_{21}(\nu) & S_{22}(\nu)
\end{array}\right),
$$

the microwave cavity was connected to the VNA via two antennas at the positions marked by 1 and 2 in Fig. 1a. Violation of $\mathcal{T}$-invariance was induced by two magnetized cylindrical NiZn ferrites (manufactured by SAMWHA, South Korea) with 
diameter $d=33 \mathrm{~mm}$ and saturation magnetization 2600 Oe. They were inserted into the cavity and magnetized by pairs of cylindrical magnets of the same diameter and of height $30 \mathrm{~mm}$ of the type N42 with coercivity 11850 Oe $(943 \mathrm{kA} / \mathrm{m})$ and magnetic field strength $B \simeq 495 \mathrm{mT}$, that were placed outside the cavity below and above the ferrites. The magnetic field $B$ induces a macroscopic magnetization $M$ of the ferrites across their cross-sections. The precession of magnetization around $B$ is taking place with the Larmor frequency $\omega_{0}=\gamma_{G} B$, where $\gamma_{G} \simeq g_{\text {eff }} 14 \mathrm{GHz} / \mathrm{T}$ and $g_{\mathrm{eff}} \simeq 2.3$ denote the gyromagnetic ratio and the Landé factor, respectively.

If the microwave frequency approaches that of the ferromagnetic resonances, then the induced violation of time reversal invariance becomes stronger. However, we observed that even for lower microwave frequencies the induced violation of time reversal invariance is quite significant. This leads to differences between $S_{12}(\nu)$ and $S_{21}(\nu)$, while for systems with preserved $\mathcal{T}$-invariance, the $\hat{S}(\nu)$ matrix is symmetric, i.e., $S_{12}(\nu)=S_{21}(\nu)$. In our experiment, the strongest effect was observed in the frequency range of $8.3-9.3 \mathrm{GHz}$. In Fig. $1 \mathrm{~b}$, we show examples for $\left|S_{12}(\nu)\right|$ (full line) and $\left|S_{21}(\nu)\right|$ (dotted line) measured in the frequency range $\nu=8.0$ $9.7 \mathrm{GHz}$ for $M=2, M=6$, and $M=12$ open channels, respectively.

\section{Enhancement factor}

For systems with partially violated $\mathcal{T}$-invariance, the enhancement factor $F_{M}(\eta, \gamma, \xi)$ is a complicated function of the openness $\eta[32]$, the Ohmic internal absorption strength $\gamma$ and the $\mathcal{T}$-invariance violation parameter $\xi$.

Such systems can be described by an ensemble of $N \times N$-dimensional random matrices composed of real symmetric and antisymmetric random matrices, respectively, interpolating between GOE for $\xi=0$ and GUE for $\xi \simeq 1$ [27]. The openness $\eta$ can be relatively easily controlled in the experiment. It depends on the number of open channels $M$ characterized by the transmission coefficients $T_{i}$, where $0<T_{i}<1$. The latter, in fact, describe the coupling of the antennas to the electric field modes inside the cavity. The openness $\eta=M T=t_{H} / t_{W}$ is defined as the ratio of two characteristic times, namely, the Heisenberg time $t_{H}=2 \pi / \Delta$ and the dwell (or the Weisskopf) time $t_{W}=1 / \Gamma_{\text {esc }}$ [31], which gives the time an incoming microwave spends inside a cavity before it escapes through one of the $M$ open channels. The Ohmic absorption strength $\gamma$ is due to absorption of the electromagnetic field in the walls of the cavity and in the ferrites. Then, the total absorption of the microwave cavity is given by $\gamma^{\text {tot }}=\eta+\gamma$.

It is commonly known that ferrite is a lossy material. Hence, it causes a degradation of both the internal absorption and the quality factor of
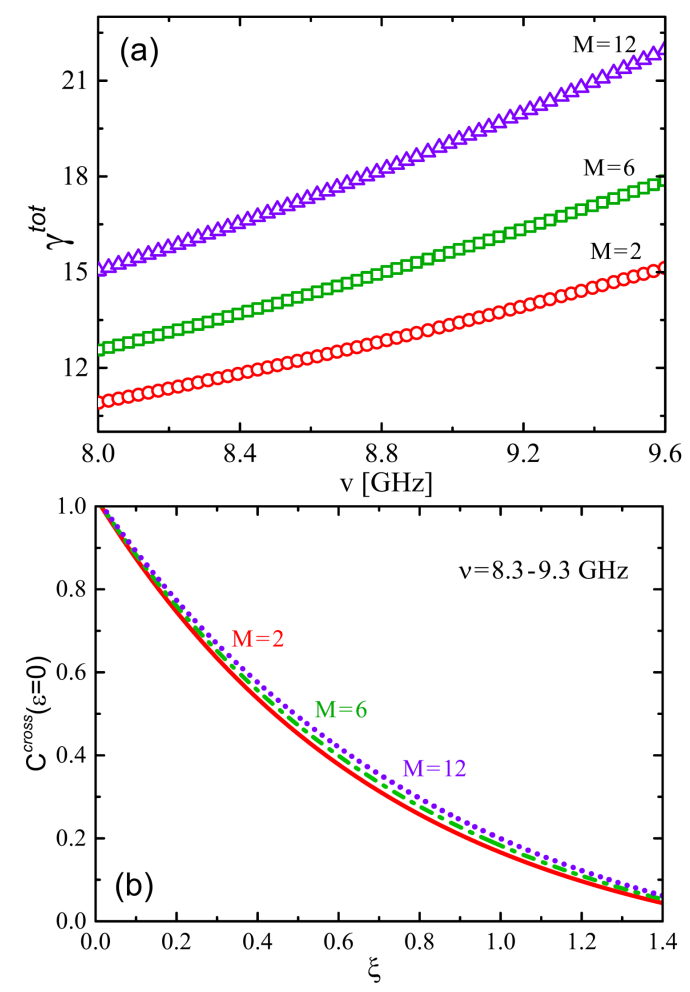

Fig. 2. (a) Total absorption width $\gamma^{\text {tot }}$ versus microwave frequency $\nu$ for $M=2$ (red circles), $M=6$ (green squares) and $M=12$ (violet triangles) channels, respectively. (b) The cross-correlation function $C^{\text {cross }}(\varepsilon=0)$ obtained from the analytical result [26], using the experimental values of $\eta$ and $\gamma$ in a frequency range $\nu=8.3-9.3 \mathrm{GHz}$ for $M=2$ (red full line), $M=6$ (green dotted-dashed line) and $M=12$ (violet dotted line) channels, respectively, versus the cross-correlation parameter $\xi$.

the system near the gyromagnetic resonance. The average transmission coefficient $T=\frac{1}{M} \sum_{i=1}^{M} T_{i}$ depends weakly on the microwave frequency and is approximately constant in a $1 \mathrm{GHz}$ frequency window. We checked that all antenna channels have similar characteristics and found that all $T_{i}$ were within $20 \%$ of each other in the frequency range from 6 to $12 \mathrm{GHz}$. In Fig. 2a the total absorption width $\gamma^{\text {tot }}$ is presented versus microwave frequency $\nu$ for $M=2$ (red circles), $M=6$ (green squares) and $M=12$ (violet triangles) channels, respectively.

To quantify the violation of $\mathcal{T}$-invariance we use the cross-correlation coefficient

$$
C^{\text {cross }}(0)=\frac{\Re\left[\left\langle S_{a b}^{f l}(\nu) S_{b a}^{f l *}(\nu)\right\rangle\right]}{\sqrt{\left\langle\left|S_{a b}^{f l}(\nu)\right|^{2}\right\rangle\left\langle\left|S_{b a}^{f l}(\nu)\right|^{2}\right\rangle}},
$$

where $C^{\text {cross }}(0)=C^{\text {cross }}(\varepsilon=0 ; \eta, \gamma, \xi)$. For systems with completely violated $\mathcal{T}$-invariance, the cross-correlation coefficient vanishes $C^{\text {cross }}(0)=0$, because both $S_{a b}$ and $S_{b a}^{*}$ are uncorrelated. In turn, $C^{\text {cross }}(0)=1$ for $\mathcal{T}$-invariant systems. In Fig. $2 \mathrm{~b}$, we show the cross-correlation function $C^{\text {cross }}(0)$ 
obtained from the analytical result [26], using the experimental values of $\eta$ and $\gamma$ in a frequency range $\nu=8.3-9.3 \mathrm{GHz}$ for $M=2$ (red full line), $M=6$ (green dotted-dashed line) and $M=12$ (violet dotted line) channels, respectively, versus the cross-correlation parameter $\xi$. The sizes of $\gamma$ and $\xi$ were determined by comparing the distributions of the experimental reflection coefficients $S_{11}$ and $S_{22}$ and the cross-correlation coefficient to analytical and numerical RMT results [26, 27, 30, 66]. Figure $2 \mathrm{~b}$ clearly shows that for a given value of the cross-correlation coefficient $C^{\text {cross }}(0)$, the value of $\xi$ increases in a function of the number of open channels $M$. Note that for a given frequency and magnetic field strength, the size of $\mathcal{T}$-invariance violation decreases with the openness of the cavity. This is expected because it depends on the electric-field intensity around the ferrite [19] which decreases with increasing $\eta$.

The elastic enhancement factor $F_{M}(\eta, \gamma, \xi)$ is given as

$$
F_{M}(\eta, \gamma, \xi)=\frac{\sqrt{C_{a a}(0 ; \eta, \gamma, \xi) C_{b b}(0 ; \eta, \gamma, \xi)}}{\sqrt{C_{a b}(0 ; \eta, \gamma, \xi) C_{b a}(0 ; \eta, \gamma, \xi)}}
$$

where $C_{a b}(0)$ denotes the $S$-matrix autocorrelation function

$$
C_{a b}(\varepsilon)=\left\langle S_{a b}^{f l}(\nu) S_{a b}^{f l *}(\nu+\varepsilon)\right\rangle
$$

at $\varepsilon=0$ [27] and $S_{a b}^{f l}(\nu)=S_{a b}(\nu)-\left\langle S_{a b}(\nu)\right\rangle$ is the fluctuating part of the two-port $S$-matrix element $S_{a b}(\nu)$. The enhancement factor was studied theoretically and investigated experimentally for systems with partially violated $\mathcal{T}$-invariance in the limit of isolated resonances $\Gamma \ll \Delta[32,34]$, of strongly overlapping ones $\Gamma \gg \Delta[47,48]$ and of weakly overlapping ones [26, 27]. It attains the following limiting values:

$$
F_{M}^{(\beta)}(\eta, \gamma) \rightarrow \begin{cases}2 \leq F_{M}^{(1)}(\eta, \gamma) \leq 3 ; & \xi=0 \\ 1 \leq F_{M}^{(2)}(\eta, \gamma) \leq 2 ; & \xi=1\end{cases}
$$

When $\mathcal{T}$-invariance is partially violated, a typical, classically chaotic quantum system is described by an RMT model interpolating between GOE and GUE. Then, in the limit of strongly overlapping resonances and of many open channels $M$, the transition from preserved to completely violated $\mathcal{T}$-invariance occurs in the range $1<F_{M}(\eta, \gamma, \xi \neq 0)<2$. In the experiment, the presence of the magnetized ferrites leads to partial violation of $\mathcal{T}$-invariance. Its strength was controlled by changing the volume of the ferrites or the strength of the external magnetic field. The largest value of $\xi=0.49$ was achieved in the frequency range of $\nu=8-9.6 \mathrm{GHz}$ to which we restrict in the following.

The experimental results for the enhancement factor $F_{M}(\eta, \gamma, \xi)$ for $M=2$ (red circles), $M=6$ (green squares) and $M=12$ (blue triangles) for $\xi=0.49$ measured in a frequency range $\nu=8-9.6 \mathrm{GHz}$ are shown in Fig. 3. The results were obtained by averaging over 100 different cavity

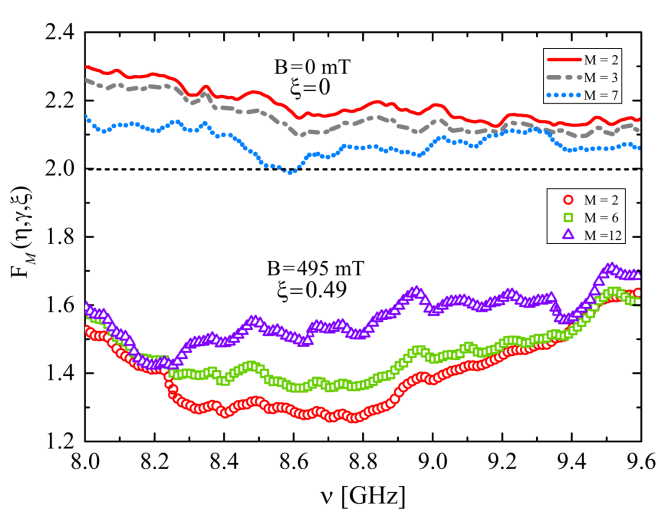

Fig. 3. The lower part of the figure shows the elastic enhancement factor calculated from the two-port scattering matrix $\hat{S}(\nu)$ measured for the microwave billiard with partially violated $\mathcal{T}$-invariance in a frequency range $\nu=8-9.6 \mathrm{GHz}$ for $M=2$ (red circles), $M=6$ (green squares), and $M=12$ (violet triangles) open channels. The black dashed line shows the RMT limit $F_{M}(\eta, \gamma, \xi=0)=2$ for very strong absorption. The upper part of the figure shows the elastic enhancement factor measured for the microwave billiard with preserved $\mathcal{T}$-invariance in a frequency range $\nu=8-9.6 \mathrm{GHz}$ for $M=2$ (red full line), $M=3$ (grey dotted-dashed line), and $M=7$ (blue dotted line) open channels.

realizations in order to reduce significant fluctuations of the enhancement factor. Each realization corresponds to a different position of the metallic scatterer which was moved alongside the vertical walls. The black dashed line shows the RMT limit $F_{M}(\eta, \gamma, \xi=0)=2$ for very strong absorption. The enhancement factor $F_{M}(\eta, \gamma, \xi)$ for $\xi=0.49$ is below $F_{M}(\eta, \gamma, \xi=0)=2$ which is impossible for a system with preserved $\mathcal{T}$-invariance. The behavior of the enhancement factor clearly indicates the presence of partial $\mathcal{T}$-invariance violation. Furthermore, the value $F_{M}(\eta, \gamma, \xi)$ depends on the number of the scattering channels. The smaller the number of channels, the lower the value of the enhancement factor $F_{M}(\eta, \gamma, \xi)$.

Moreover, for a fixed $M$ the enhancement factor $F_{M}(\eta, \gamma, \xi)$ increases with increasing frequency and, thus, with increasing Ohmic absorption strength $\gamma=\gamma^{\text {tot }}-M T$. This is distinct from the case of preserved $\mathcal{T}$-invariance where for fixed $M$ the enhancement factor $F_{M}(\eta, \gamma, \xi=0)$ decreases with increasing $\gamma$. This phenomenon is clearly seen in the upper part of Fig. 3, where we show for comparison the enhancement factor $F_{M}(\eta, \gamma, \xi=0)$ for the same microwave billiard but with preserved $\mathcal{T}$-invariance. The elastic enhancement factor was measured in a frequency range $\nu=8-9.6 \mathrm{GHz}$ for $M=2$ (red full line), $M=3$ (grey dotted-dashed line), and $M=7$ (blue dotted line) open channels. For a fixed $M$, it decreases with the microwave frequency, i.e., with increasing absorption strength $\gamma$. 


\section{Conclusion}

We investigated partial $\mathcal{T}$-invariance violation in a fully chaotic quarter-bow-tie microwave billiard by utilizing properties of the elastic enhancement factor $F_{M}(\eta, \gamma, \xi)$. The experimental and numerical results for the enhancement factor corroborate the visible crossover from orthogonal to unitary symmetry (GOE $\rightarrow$ GUE). Ferromagnetic resonances were induced for $\nu>8 \mathrm{GHz}$ by magnetized ferrites inside the cavity. In this range, the enhancement factor lies below the lower limit $F_{M}(\eta, \gamma, \xi=0)=2$ predicted for the orthogonal symmetry class. The dependence of the strength of $\mathcal{T}$-invariance violation appears to be a function of the openness $\eta$ determined by the number of scattering channels $M$, where we found out that the smaller the number of channels, the lower the value of the enhancement factor $F_{M}(\eta, \gamma, \xi)$. It is important to point out that for a fixed $M$, the enhancement factor $F_{M}(\eta, \gamma, \xi)$ increases with increasing Ohmic absorption strength $\gamma$ which is distinct from the case of preserved $\mathcal{T}$-invariance where the enhancement factor $F_{M}(\eta, \gamma, \xi=0)$ decreases with increasing $\gamma$.

\section{Acknowledgments}

This work was partly supported by the National Science Centre, Poland, grant No. UMO2018/30/Q/ST2/00324. B.D. thanks the National Natural Science Foundation of China for financial support through Grants Nos. 11775100, 11961131009 and 12047501 . Supported by the 111 Project under Grant No. B20063.

\section{References}

[1] O. Bohigas, M.J. Giannoni, C. Schmit, Phys. Rev. Lett. 52, 1 (1984).

[2] M.L. Mehta, Random Matrices, 3rd ed., Elsevier, Amsterdam 2004.

[3] L. Vina, M. Potemski, W. Wang, Phys.Usp. 41, 153 (1998).

[4] T. Zimmermann, H. Köppel, L.S. Cederbaum, G. Persch, W. Demtröder, Phys. Rev. Lett. 61, 3 (1988).

[5] H.-J. Stöckmann, J. Stein, Phys. Rev. Lett. 64, 2215 (1990).

[6] O. Hul, S. Bauch, P. Pakoński, N. Savytskyy, K. Życzkowski, L. Sirko, Phys. Rev. E 69, 056205 (2004).

[7] B. Dietz, V. Yunko, M. Białous, S. Bauch, M. Ławniczak, L. Sirko, Phys. Rev. E 95, 052202 (2017).

[8] K. Sacha, J. Zakrzewski, D. Delande, Phys. Rev. Lett. 83, 2922 (1999).

[9] L.A. Ponomarenko, F. Schedlin, M.I. Katsnelson, R. Yang, E.W. Hill, K.S. Novoselov, A.K. Geim, Science 320, 356 (2008).
[10] M. Aßmann, J. Thewes, D. Fröhlich, M. Bayer, Nature Mater. 15, 741 (2016).

[11] J.B. French, V.K.B. Kota, A. Pandey, S. Tomsovic, Phys. Rev. Lett. 54, 2313 (1985).

[12] G.E. Mitchell, A. Richter, H.A. Weidenmüller, Rev. Mod. Phys. 82, 2845 (2010).

[13] P. So, S.M. Anlage, E. Ott, R.N. Oerter, Phys. Rev. Lett. 74, 2662 (1995).

[14] U. Stoffregen, J. Stein, H.-J. Stöckmann, M. Kuś, F. Haake, Phys. Rev. Lett. 74, 2666 (1995).

[15] M. Allgaier, S. Gehler, S. Barkhofen, H.-J. Stöckmann, U. Kuhl, Phys. Rev. E 89, 022925 (2014).

[16] M. Białous, V. Yunko, S. Bauch, M. Ławniczak, B. Dietz, L. Sirko, Phys. Rev. Lett. 117, 144101 (2016).

[17] G. Lenz, K. Życzkowski, J. Phys. A 25, 5539 (1992).

[18] S. Schierenberg, F. Bruckmann, T. Wetting, Phys. Rev. E 85, 061130 (2012).

[19] B. Dietz, T. Klaus, M. Miski-Oglu, A. Richter, M. Wunderle, Phys. Rev. Lett 123, 174101 (2019).

[20] M. Białous, B. Dietz, L. Sirko, Phys. Rev. E 102, 042206 (2020).

[21] F. Haake, Quantum Signatures of Chaos, 3rd ed., Springer Series in Synergetics, Springer, Heidelberg 2010.

[22] G. Lenz, Ph.D. Thesis, Universität Essen, 1992.

[23] T. Guhr, Ann. Phys. 250, 145 (1996).

[24] M.V. Berry, M. Tabor, Proc. R. Soc. Lond. A 356, 375 (1977).

[25] M. Białous, V. Yunko, S. Bauch, M. Ławniczak, B. Dietz, L. Sirko, Phys. Rev. E 94, 042211 (2016).

[26] B. Dietz, T. Friedrich, H.L. Harney, M. Miski-Oglu, A. Richter, F. Schäfer, J. Verbaarschot, H.A. Weidenmüller, Phys. Rev. Lett. 103, 064101 (2009).

[27] B. Dietz, T. Friedrich, H.L. Harney, M. Miski-Oglu, A. Richter, F. Schäfer, H.A. Weidenmüller, Phys. Rev. E 81, 036205 (2010).

[28] P.A. Moldauer, Phys. Rev. 135B, 642 (1964).

[29] J.J.M. Verbaarschot, Ann. Phys. 168, 368 (1986).

[30] Y.V. Fyodorov, D.V. Savin, H.J. Sommers, J. Phys. A Math. Gen. 38, 10731 (2005).

[31] Y.A. Kharkov, V.V. Sokolov, Phys. Lett. B 718, 1562 (2013).

[32] V.V. Sokolov, O.V. Zhirov, Acta Phys. Pol. A 128, 990 (2015). 
[33] J.J.M. Verbaaschot, H.A. Weidenmüller, M.R. Zirnbauer, Phys. Rep. 129367 (1985).

[34] J.-H. Yeh, Z. Drikas, J. Gil Gil, S. Hong, B.T. Taddese, E. Ott, T.M. Antonsen, T. Andreadis, S.M. Anlage, Acta Phys. Pol. A 124, 1045 (2013).

[35] X. Zheng, S. Hemmady, T.M. Antonsen, Jr., S.M. Anlage, E. Ott, Phys. Rev. E 73 046208 (2006).

[36] S. Sridhar, A. Kudrolli, Phys. Rev. Lett. 72, 2175 (1994).

[37] L. Sirko, P.M. Koch, R. Blümel, Phys. Rev. Lett. 78, 2940 (1997).

[38] Y. Hlushchuk, A. Kohler, Sz. Bauch, L. Sirko, R. Blümel, M. Barth, H.-J. Stöckmann, Phys. Rev. E 61, 366 (2000).

[39] Y. Hlushchuk, A. Błędowski, N. Savytskyy, L. Sirko, Phys. Scr. 64, 192 (2001).

[40] R. Blümel, P.M. Koch, L. Sirko, Found. Phys. 31, 269 (2001).

[41] A. Dhar, D.M. Rao, U. Shankar, S. Sridhar, Phys. Rev. E 68, 026208 (2003).

[42] S. Hemmady, X. Zheng, E. Ott, T.M. Antonsen, S.M. Anlage, Phys. Rev. Lett. 94, 014102 (2005).

[43] O. Hul, O. Tymoshchuk, S. Bauch, P.M. Koch, L. Sirko, J. Phys. A 38, 10489 (2005).

[44] S. Hemmady, X. Zheng, J. Hart, T.M. Antonsen, Jr., E. Ott, S.M. Anlage, Phys. Rev. E 74, 036213 (2006).

[45] B. Dietz, A. Richter, Chaos 25, 097601 (2015).

[46] M. Białous, B. Dietz, L. Sirko, Phys. Rev. E 100, 012210 (2019).

[47] M. Ławniczak, S. Bauch, O. Hul, L. Sirko, Phys. Rev. E 81, 046204 (2010).

[48] M. Ławniczak, L. Sirko, Sci. Rep. 9, 5630 (2019).

[49] A. Rehemanjiang, M. Allgaier, C.H. Joyner, S. Müller, M. Sieber, U. Kuhl, H.-J. Stöckmann, Phys. Rev. Lett. 117, 064101 (2016).
[50] M. Ławniczak, J. Lipovský, L. Sirko, Phys. Rev. Lett 122, 140503 (2019).

[51] J. Lu, J. Che, X. Zhang, B. Dietz, Phys. Rev. E 102, 022309 (2020).

[52] V. Yunko, M. Białous, L. Sirko, Phys. Rev. E 102, 012210 (2020).

[53] R. Blümel, A. Buchleitner, R. Graham, L. Sirko, U. Smilansky, H. Walther, Phys. Rev. A 44, 4521 (1991).

[54] R.V. Jensen, S.M. Susskind, M.M. Sanders, Phys. Rep. 201, 1 (1991).

[55] M. Bellermann, T. Bergemann, A. Haffmann, P.M. Koch, L. Sirko, Phys. Rev. A 46, 5836 (1992).

[56] L. Sirko, S. Yoakum, A. Haffmans, P.M. Koch, Phys. Rev. A 47, R782 (1993).

[57] A. Buchleitner, D. Delande, Phys. Rev. Lett. 71, 3633 (1993).

[58] L. Sirko, M.R.W. Bellermann, A. Haffmans, P.M. Koch, D. Richards, Phys. Rev. Lett. 71, 2895 (1993).

[59] J.E. Bayfield, S.-Y. Luie, L.C. Perotti, M.P. Skrzypkowski, Physica D Nonlin. Phenom. 83, 46 (1995).

[60] L. Sirko, P.M. Koch, Appl. Phys. B 60, S195 (1995).

[61] L. Sirko, A. Haffmans, M.R.W. Bellermann, P.M. Koch, Europhys. Lett. 33, 181 (1996).

[62] J. Bayfield, Lal Pinnaduwage, J. Phys. B 18, L49 (1999).

[63] L. Sirko, S.A. Zelazny, P.M. Koch, Phys. Rev. Lett. 87, 043002 (2001).

[64] L. Sirko, P.M. Koch, Phys. Rev. Lett. 89, 274101 (2002).

[65] A. Arakelyan, J. Nunkaew, T.F. Gallagher, Phys. Rev. A 94, 053416 (2016).

[66] S. Kumar, A. Nock, H.-J. Sommers, T. Guhr, B. Dietz, M. Miski-Oglu, A. Richter, F. Schäfer, Phys. Rev. Lett. 111, 030403 (2013). 